\title{
Genomoviridae: a new family of widespread single-stranded DNA viruses
}

\author{
Mart Krupovic $^{1}$ (D) Said A. Ghabrial ${ }^{2} \cdot$ Daohong Jiang $^{3} \cdot$ Arvind Varsani $^{4,5,6}$
}

Received: 2 May 2016/Accepted: 17 June 2016/Published online: 24 June 2016

(C) Springer-Verlag Wien 2016

\begin{abstract}
Here, we introduce a new family of eukaryoteinfecting single-stranded (ss) DNA viruses that was created recently by the International Committee on Taxonomy of Viruses (ICTV). The family, named Genomoviridae, contains a single genus, Gemycircularvirus, which currently has one recognized virus species, Sclerotinia gemycircularvirus 1. Sclerotinia sclerotiorum hypovirulence-associated DNA virus 1 (SsHADV-1) is currently the sole representative isolate of the family; however, a great number of SsHADV1-like ssDNA virus genomes has been sequenced from various environmental, plant- and animal-associated samples, indicating that members of family Genomoviridae are widespread and abundant in the environment.
\end{abstract}

Mart Krupovic

krupovic@pasteur.fr

$\square$ Arvind Varsani

arvind.varsani@canterbury.ac.nz

1 Unité de Biologie Moléculaire du Gène chez les Extrêmophiles, Department of Microbiology, Institut Pasteur, Paris, France

2 Plant Pathology Department, University of Kentucky, Lexington, KY 40546, USA

3 State Key Lab of Agricultural Microbiology, Huazhong Agricultural University, Wuhan, Hubei, People's Republic of China

4 School of Biological Sciences and Biomolecular Interaction Centre, University of Canterbury,

Private Bag 4800, Christchurch, New Zealand

5 Structural Biology Research Unit, Division of Medical Biochemistry, Department of Clinical Laboratory Sciences, University of Cape Town, Cape Town, South Africa

6 Department of Plant Pathology and Emerging Pathogens Institute, University of Florida, Gainesville, FL, USA
Viruses with single-stranded DNA (ssDNA) genomes infect hosts in all three domains of life and include economically, medically, and environmentally important viral pathogens. Until recently, ssDNA viruses were classified into 10 different taxa-nine families and one genus not assigned to a family $[14,15]$. In 2016, the International Committee on Taxonomy of Viruses (ICTV) created two new families for classification of ssDNA viruses, Pleolipoviridae and Genomoviridae [1]. The family Pleolipoviridae includes viruses infecting hyperhalophilic archaea, and it has recently been described elsewhere [25]. Here, we introduce the family Genomoviridae and provide a short overview of the properties of Sclerotinia sclerotiorum hypovirulence-associated DNA virus 1 (SsHADV1 ), the only cultivated member of the new family, and putative SsHADV-1-like viruses.

SsHADV-1 is the first—and thus far the only-ssDNA virus known to infect fungi (all other fungal viruses have RNA genomes) [10, 33]. SsHADV-1 was isolated from a plant-pathogenic fungus, Sclerotinia sclerotiorum [34]. Another unique property of SsHADV-1 that has not been described for other fungal viruses is its ability to establish infection when applied extracellularly in the form of purified viral particles [35]. Virions can infect the hyphae of virus-free $S$. sclerotiorum directly when applied to hyphae or sprayed on leaves of Arabidopsis thaliana and Brassica napus infected with S. sclerotiorum. When applied to S. sclerotiorum-infected leaves, the virus can suppress the development of $S$. sclerotiorum-induced lesions. SsHADV-1 has a narrow host range and, in addition to $S$. sclerotiorum, can infect the related species $S$. minor and $S$. nivalis, but not other relatively closely related fungi, such as Botrytis cinerea [35]. Furthermore, SsHADV-1 has been identified in New Zealand and the USA in environmental samples and insects; however, this 
is not surprising given the near global distribution of $S$. sclerotiorum [6, 11].

SsHADV-1 virions are non-enveloped, isometric, 20-22 $\mathrm{nm}$ in diameter, and constructed from one capsid protein (CP) [34]. The genome is a circular ssDNA molecule of 2,166 nucleotides and contains two genes - for CP and replication initiation protein (Rep) (Fig. 1). The prediction of the $c p$ gene has been validated by $\mathrm{N}$-terminal sequencing of the $\mathrm{CP}$ purified from the virions. The large intergenic region contains a potential stem-loop structure with a nonanucleotide (TAATATTAT) motif at its apex, which is likely to be important for rolling-circle replication. The CP of SsHADV-1 is not recognizably similar to the corresponding proteins from viruses in other taxa.

Although SsHADV-1 remains the only isolated member of the group, genomes of more than 100 SsHADV-1-like putative viruses have been reported (see Table 1). These genomes have been sequenced from different environmental samples, and many were identified associated with plant material as well as various animal-associated samples, including cerebrospinal fluid and blood of humans (Table 1 and references therein). Even though the hosts of these putative viruses remain unknown, their diversity suggests that SsHADV-1-like viruses are abundant and widespread in the environment.

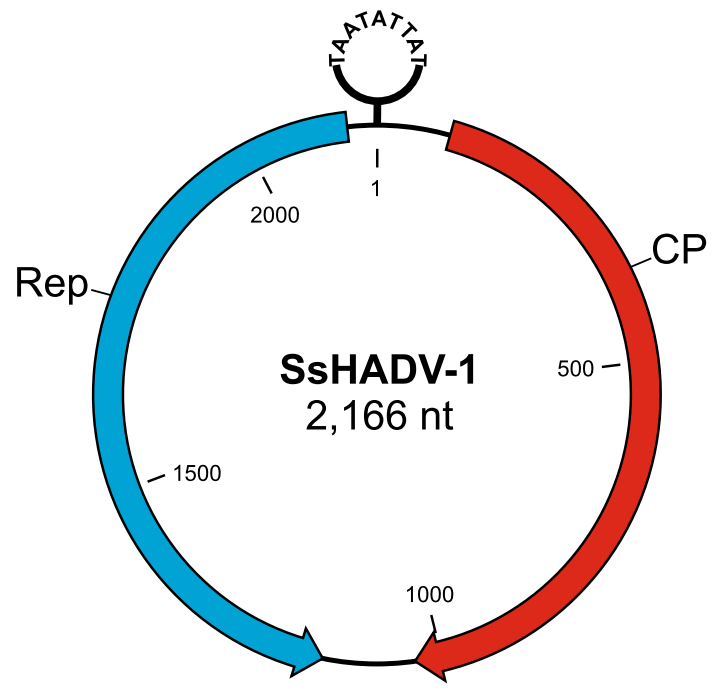

Fig. 1 Genome map of SsHADV-1. Genes encoding the replicationinitiation protein (Rep) and capsid protein $(\mathrm{CP})$ are shown with blue and red arrows, respectively. The position of the nonanucleotide (TAATATTAT) at the apex of a potential stem-loop structure is also indicated 3
All putative SsHADV-1-like viruses encode homologous Rep and $\mathrm{CP}$, and in phylogenetic analyses form monophyletic clades with SsHADV-1 (Figures 2 and 3). Their genomes are of similar size, in the range of 2,089-2,290 nucleotides. Structural and genomic features of SsHADV-1 differ considerably from those of all other currently classified viruses. The Rep of SsHADV-1 is most closely related to the corresponding proteins of members of the family Geminiviridae. It shares with geminiviral proteins two conserved domains, namely geminivirus Rep catalytic domain (Gemini_AL1; PF00799) and geminivirus Rep protein central domain (Gemini_AL1_M; PF08283), with conserved motifs for rolling-circle replication. Notably, similar to some geminiviruses [31], certain SsHADV-1-like viruses also contain introns within their Rep-encoding genes $[6,7,12,13,18$, 19, 26-28, 30]. However, Rep-based phylogenetic analysis shows that SsHADV-1 and other related putative viruses form a well-supported, monophyletic clade, which branches as a sister group to geminiviruses (Fig. 2). Unlike the Rep, the CP of SsHADV-1 (and related viruses) does not display any recognizable sequence similarity to proteins of geminiviruses or any other group of known viruses. Furthermore, all geminiviruses possess distinctive geminate virions constructed from two incomplete $T=1$ icosahedra [2, 36], whereas the virion of SsHADV-1 is isometric [34]. Finally, the number of genes and size of the genome differ considerably between SsHADV-1-like viruses and geminiviruses. In particular, all putative SsHADV-1-like viruses lack the movement protein, which is essential for the plant geminiviruses.

In recognition of the unique features described above, SsHADV-1 has been classified into the species Sclerotinia gemycircularvirus 1 within the new genus Gemycircularvirus (Gemini-like myco-infecting circular virus) [26] within the new family Genomoviridae (sigil: $G e-$ for geminivirus-like, nomo- for no movement protein).

Although Genomoviridae currently includes only a single representative, new members, possibly including uncultivated viruses, are expected to be added to the family in the near future. Based on the available genetic data and phylogenetic analyses (Figures 2 and 3), it is already clear that many new genera will have to be introduced to adequately reflect the diversity of SsHADV-1-like viruses. 
Table 1 Genome sequences of putative SsHADV-1-like viruses

\begin{tabular}{|c|c|c|c|c|c|c|c|}
\hline $\begin{array}{l}\text { GenBank } \\
\text { accession } \\
\text { no. }\end{array}$ & Virus/putative virus name & Acronym & Country & Isolation source & $\begin{array}{l}\text { Common } \\
\text { name }\end{array}$ & Sample type & Reference \\
\hline GQ365709 & $\begin{array}{l}\text { Sclerotinia sclerotiorum } \\
\text { hypovirulence associated } \\
\text { DNA virus } 1\end{array}$ & SsHADV-1 & China & $\begin{array}{l}\text { Sclerotinia } \\
\text { sclerotiorum }\end{array}$ & White mold & $\begin{array}{l}\text { Mycelial } \\
\text { samples }\end{array}$ & {$[34]$} \\
\hline KF268025 & $\begin{array}{l}\text { Sclerotinia sclerotiorum } \\
\text { hypovirulence associated } \\
\text { DNA virus } 1\end{array}$ & SsHADV-1 & $\begin{array}{l}\text { New } \\
\text { Zealand }\end{array}$ & River Sediments & - & $\begin{array}{l}\text { River } \\
\text { Sediments }\end{array}$ & {$[11]$} \\
\hline KF268026 & $\begin{array}{l}\text { Sclerotinia sclerotiorum } \\
\text { hypovirulence associated } \\
\text { DNA virus } 1\end{array}$ & SsHADV-1 & $\begin{array}{l}\text { New } \\
\text { Zealand }\end{array}$ & River Sediments & - & $\begin{array}{l}\text { River } \\
\text { Sediments }\end{array}$ & [11] \\
\hline KF268027 & $\begin{array}{l}\text { Sclerotinia sclerotiorum } \\
\text { hypovirulence associated } \\
\text { DNA virus } 1\end{array}$ & SsHADV-1 & $\begin{array}{l}\text { New } \\
\text { Zealand }\end{array}$ & River Sediments & - & $\begin{array}{l}\text { River } \\
\text { Sediments }\end{array}$ & {$[11]$} \\
\hline KF268028 & $\begin{array}{l}\text { Sclerotinia sclerotiorum } \\
\text { hypovirulence associated } \\
\text { DNA virus } 1\end{array}$ & SsHADV-1 & $\begin{array}{l}\text { New } \\
\text { Zealand }\end{array}$ & River Sediments & - & $\begin{array}{l}\text { River } \\
\text { Sediments }\end{array}$ & {$[11]$} \\
\hline KM598382 & $\begin{array}{l}\text { Sclerotinia sclerotiorum } \\
\text { hypovirulence associated } \\
\text { DNA virus } 1\end{array}$ & SsHADV-1 & USA & $\begin{array}{l}\text { Ischnura } \\
\text { ramburii }\end{array}$ & Damselfly & Abdomen & [6] \\
\hline KM598383 & $\begin{array}{l}\text { Sclerotinia sclerotiorum } \\
\text { hypovirulence associated } \\
\text { DNA virus } 1\end{array}$ & SsHADV-1 & USA & $\begin{array}{l}\text { Erythemis } \\
\quad \text { simplicicollis }\end{array}$ & Dragonfly & Abdomen & [6] \\
\hline KM598384 & $\begin{array}{l}\text { Sclerotinia sclerotiorum } \\
\text { hypovirulence associated } \\
\text { DNA virus } 1\end{array}$ & SsHADV-1 & USA & $\begin{array}{l}\text { Pantala } \\
\text { hymenaea }\end{array}$ & Dragonfly & Abdomen & [6] \\
\hline HQ335086 & $\begin{array}{l}\text { Mosquito VEM virus } \\
\text { SDBVL G }\end{array}$ & MVemV & USA & $\begin{array}{l}\text { Culex } \\
\text { erythrothorax }\end{array}$ & Mosquito & $\begin{array}{l}\text { Mosquito } \\
\text { samples }\end{array}$ & {$[22]$} \\
\hline JN704610 & Meles meles fecal virus & $\mathrm{MmFV}$ & Netherlands & Meles meles & $\begin{array}{c}\text { European } \\
\text { badger }\end{array}$ & Rectal swab & {$[30]$} \\
\hline JQ412057 & $\begin{array}{l}\text { Cassava associated circular } \\
\text { DNA virus }\end{array}$ & $\mathrm{CasCV}$ & Ghana & $\begin{array}{l}\text { Manihot } \\
\text { esculenta }\end{array}$ & Cassava & Leaf & [5] \\
\hline JX185428 & $\begin{array}{l}\text { Dragonfly-associated circular } \\
\text { virus } 3\end{array}$ & DfasCV-3 & Tonga & $\begin{array}{l}\text { Pantala } \\
\text { flavescens }\end{array}$ & Dragonfly & Abdomen & [26] \\
\hline JX185429 & $\begin{array}{l}\text { Dragonfly-associated circular } \\
\text { virus } 2\end{array}$ & DfasCV-2 & USA & $\begin{array}{l}\text { Erythemis } \\
\quad \text { simplicicollis }\end{array}$ & Dragonfly & Abdomen & [26] \\
\hline JX185430 & $\begin{array}{l}\text { Dragonfly-associated circular } \\
\text { virus } 1\end{array}$ & DfasCV-1 & USA & $\begin{array}{l}\text { Miathyria } \\
\text { marcella }\end{array}$ & Dragonfly & Abdomen & [26] \\
\hline KF371630 & $\begin{array}{l}\text { Faecal-associated } \\
\text { gemycircularvirus-12 }\end{array}$ & FaGmV-12 & $\begin{array}{l}\text { New } \\
\text { Zealand }\end{array}$ & Struthio camelus & Ostrich & Faeces & [27] \\
\hline KF371631 & $\begin{array}{l}\text { Faecal-associated } \\
\text { gemycircularvirus-11 }\end{array}$ & FaGmV-11 & $\begin{array}{l}\text { New } \\
\text { Zealand }\end{array}$ & $\begin{array}{l}\text { Oryctolagus } \\
\text { cuniculus }\end{array}$ & Rabbit & Faeces & [27] \\
\hline KF371632 & $\begin{array}{l}\text { Faecal-associated } \\
\text { gemycircularvirus-10 }\end{array}$ & FaGmV-10 & $\begin{array}{l}\text { New } \\
\text { Zealand }\end{array}$ & Sturnus vulgaris & $\begin{array}{r}\text { European } \\
\text { starling }\end{array}$ & Faeces & [27] \\
\hline KF371633 & $\begin{array}{l}\text { Faecal-associated } \\
\text { gemycircularvirus-9 }\end{array}$ & FaGmV-9 & $\begin{array}{l}\text { New } \\
\text { Zealand }\end{array}$ & Turdus merula & Blackbird & Faeces & [27] \\
\hline KF371634 & $\begin{array}{l}\text { Faecal-associated } \\
\text { gemycircularvirus-8 }\end{array}$ & $\mathrm{FaGmV}-8$ & $\begin{array}{l}\text { New } \\
\text { Zealand }\end{array}$ & Petroica traversi & $\begin{array}{l}\text { Chatham } \\
\text { Island black } \\
\text { robin }\end{array}$ & Faeces & [27] \\
\hline KF371635 & $\begin{array}{l}\text { Faecal-associated } \\
\text { gemycircularvirus-7 }\end{array}$ & FaGmV-7 & $\begin{array}{l}\text { New } \\
\text { Zealand }\end{array}$ & $\begin{array}{l}\text { Anas } \\
\quad \text { platyrhynchos }\end{array}$ & Mallard duck & Faeces & [27] \\
\hline KF371636 & $\begin{array}{l}\text { Faecal-associated } \\
\text { gemycircularvirus-6 }\end{array}$ & FaGmV-6 & $\begin{array}{l}\text { New } \\
\text { Zealand }\end{array}$ & $\begin{array}{l}\text { Gerygone } \\
\text { albofrontata }\end{array}$ & $\begin{array}{l}\text { Chatham } \\
\text { Island } \\
\text { warbler }\end{array}$ & Faeces & [27] \\
\hline KF371637 & $\begin{array}{l}\text { Faecal-associated } \\
\text { gemycircularvirus-5 }\end{array}$ & FaGmV-5 & $\begin{array}{l}\text { New } \\
\text { Zealand }\end{array}$ & $\begin{array}{l}\text { Gerygone } \\
\text { albofrontata }\end{array}$ & $\begin{array}{l}\text { Chatham } \\
\text { Island } \\
\text { warbler }\end{array}$ & Faeces & [27] \\
\hline
\end{tabular}


Table 1 continued

\begin{tabular}{|c|c|c|c|c|c|c|c|}
\hline $\begin{array}{l}\text { GenBank } \\
\text { accession } \\
\text { no. }\end{array}$ & Virus/putative virus name & Acronym & Country & Isolation source & $\begin{array}{l}\text { Common } \\
\text { name }\end{array}$ & Sample type & Reference \\
\hline KF371638 & $\begin{array}{l}\text { Faecal-associated } \\
\text { gemycircularvirus-4 }\end{array}$ & $\mathrm{FaGmV}-4$ & $\begin{array}{l}\text { New } \\
\text { Zealand }\end{array}$ & $\begin{array}{l}\text { Arctocephalus } \\
\text { forsteri }\end{array}$ & $\begin{array}{l}\text { New Zealand } \\
\text { fur seal }\end{array}$ & Faeces & [27] \\
\hline KF371639 & $\begin{array}{l}\text { Faecal-associated } \\
\text { gemycircularvirus-3 }\end{array}$ & $\mathrm{FaGmV}-3$ & $\begin{array}{l}\text { New } \\
\text { Zealand }\end{array}$ & $\begin{array}{l}\text { Gerygone } \\
\text { albofrontata }\end{array}$ & $\begin{array}{c}\text { Chatham } \\
\text { Island } \\
\text { warbler }\end{array}$ & Faeces & [27] \\
\hline KF371640 & $\begin{array}{l}\text { Faecal-associated } \\
\text { gemycircularvirus-2 }\end{array}$ & FaGmV-2 & $\begin{array}{l}\text { New } \\
\text { Zealand }\end{array}$ & Sus scrofa & Domestic pig & Faeces & [27] \\
\hline KF371641 & $\begin{array}{l}\text { Faecal-associated } \\
\text { gemycircularvirus-1c }\end{array}$ & FaGmV-1c & $\begin{array}{l}\text { New } \\
\text { Zealand }\end{array}$ & Turdus merula & Blackbird & Faeces & {$[27]$} \\
\hline KF371642 & $\begin{array}{l}\text { Faecal-associated } \\
\text { gemycircularvirus-1b }\end{array}$ & FaGmV-1b & $\begin{array}{l}\text { New } \\
\text { Zealand }\end{array}$ & Turdus merula & Blackbird & Faeces & [27] \\
\hline KF371643 & $\begin{array}{l}\text { Faecal-associated } \\
\text { gemycircularvirus-1a }\end{array}$ & FaGmV-1a & $\begin{array}{l}\text { New } \\
\text { Zealand }\end{array}$ & Ovis aries & Sheep & Faeces & {$[27]$} \\
\hline KF413620 & $\begin{array}{l}\text { Hypericum japonicum } \\
\text { associated circular DNA virus }\end{array}$ & HJasCV & Viet Nam & $\begin{array}{l}\text { Hypericum } \\
\text { japonicum }\end{array}$ & Hypericum & Leaf & [7] \\
\hline KJ413144 & $\begin{array}{l}\text { Human genital-associated } \\
\text { circular DNA virus-1 }\end{array}$ & HuGaGmC349 & $\begin{array}{l}\text { South } \\
\text { Africa }\end{array}$ & Homo sapiens & Human & $\begin{array}{r}\text { Cervical } \\
\text { sample }\end{array}$ & unpublished \\
\hline KJ547634 & $\begin{array}{l}\text { Sewage-associated } \\
\text { gemycircularvirus-4 }\end{array}$ & $\mathrm{SaGmV}-4$ & $\begin{array}{l}\text { New } \\
\text { Zealand }\end{array}$ & $\begin{array}{l}\text { Sewage } \\
\text { oxidation pond }\end{array}$ & - & Sewage & {$[12]$} \\
\hline KJ547635 & $\begin{array}{l}\text { Sewage-associated } \\
\text { gemycircularvirus- } 5\end{array}$ & SaGmV-5 & $\begin{array}{l}\text { New } \\
\text { Zealand }\end{array}$ & $\begin{array}{l}\text { Sewage } \\
\text { oxidation pond }\end{array}$ & - & Sewage & {$[12]$} \\
\hline KJ547636 & $\begin{array}{l}\text { Sewage-associated } \\
\text { gemycircularvirus-6 }\end{array}$ & SaGmV-6 & $\begin{array}{l}\text { New } \\
\text { Zealand }\end{array}$ & $\begin{array}{l}\text { Sewage } \\
\text { oxidation pond }\end{array}$ & - & Sewage & {$[12]$} \\
\hline KJ547637 & $\begin{array}{l}\text { Sewage-associated } \\
\text { gemycircularvirus- } 7 \mathrm{a}\end{array}$ & SaGmV-7a & $\begin{array}{l}\text { New } \\
\text { Zealand }\end{array}$ & $\begin{array}{l}\text { Sewage } \\
\text { oxidation pond }\end{array}$ & - & Sewage & {$[12]$} \\
\hline KJ547638 & $\begin{array}{l}\text { Sewage-associated } \\
\text { gemycircularvirus-8 }\end{array}$ & $\mathrm{SaGmV}-8$ & $\begin{array}{l}\text { New } \\
\text { Zealand }\end{array}$ & $\begin{array}{l}\text { Sewage } \\
\text { oxidation pond }\end{array}$ & - & Sewage & {$[12]$} \\
\hline KJ547639 & $\begin{array}{l}\text { Sewage-associated } \\
\text { gemycircularvirus-9 }\end{array}$ & SaGmV-9 & $\begin{array}{l}\text { New } \\
\text { Zealand }\end{array}$ & $\begin{array}{l}\text { Sewage } \\
\text { oxidation pond }\end{array}$ & - & Sewage & {$[12]$} \\
\hline KJ547640 & $\begin{array}{l}\text { Sewage-associated } \\
\text { gemycircularvirus-7b }\end{array}$ & SaGmV-7b & $\begin{array}{l}\text { New } \\
\text { Zealand }\end{array}$ & $\begin{array}{l}\text { Sewage } \\
\text { oxidation pond }\end{array}$ & - & Sewage & {$[12]$} \\
\hline KJ547641 & $\begin{array}{l}\text { Sewage-associated } \\
\text { gemycircularvirus-11 }\end{array}$ & SaGmV-11 & $\begin{array}{l}\text { New } \\
\text { Zealand }\end{array}$ & $\begin{array}{l}\text { Sewage } \\
\text { oxidation pond }\end{array}$ & - & Sewage & [12] \\
\hline KJ547642 & $\begin{array}{l}\text { Sewage-associated } \\
\text { gemycircularvirus-2 }\end{array}$ & SaGmV-2 & $\begin{array}{l}\text { New } \\
\text { Zealand }\end{array}$ & $\begin{array}{l}\text { Sewage } \\
\text { oxidation pond }\end{array}$ & - & Sewage & {$[12]$} \\
\hline KJ547643 & $\begin{array}{l}\text { Sewage-associated } \\
\text { gemycircularvirus-3 }\end{array}$ & SaGmV-3 & $\begin{array}{l}\text { New } \\
\text { Zealand }\end{array}$ & $\begin{array}{l}\text { Sewage } \\
\text { oxidation pond }\end{array}$ & - & Sewage & {$[12]$} \\
\hline KJ547644 & $\begin{array}{l}\text { Sewage-associated } \\
\text { gemycircularvirus-10a }\end{array}$ & $\mathrm{SaGmV}-10 \mathrm{a}$ & $\begin{array}{l}\text { New } \\
\text { Zealand }\end{array}$ & $\begin{array}{l}\text { Sewage } \\
\text { oxidation pond }\end{array}$ & - & Sewage & [12] \\
\hline KJ547645 & $\begin{array}{l}\text { Sewage-associated } \\
\text { gemycircularvirus-10b }\end{array}$ & SaGmV-10b & $\begin{array}{l}\text { New } \\
\text { Zealand }\end{array}$ & $\begin{array}{l}\text { Sewage } \\
\text { oxidation pond }\end{array}$ & - & Sewage & [12] \\
\hline KJ641719 & $\begin{array}{l}\text { Bat gemycircularvirus } 23 \\
\text { GD2012 }\end{array}$ & $\begin{array}{l}\text { BtMf-CV-23 } \\
\text { GD2012 }\end{array}$ & China & $\begin{array}{l}\text { Miniopterus } \\
\text { fuliginosus }\end{array}$ & Bat & $\begin{array}{l}\text { Pharyngeal \& } \\
\text { rectal swabs }\end{array}$ & {$[32]$} \\
\hline KJ641726 & $\begin{array}{l}\text { Bat gemycircularvirus } 8 \\
\text { NM2013 }\end{array}$ & $\begin{array}{l}\text { BtRf-CV-8 } \\
\text { NM2013 }\end{array}$ & China & $\begin{array}{l}\text { Rhinolophus } \\
\text { ferrumequinum }\end{array}$ & Bat & $\begin{array}{l}\text { Pharyngeal \& } \\
\text { rectal swabs }\end{array}$ & {$[32]$} \\
\hline KJ641737 & $\begin{array}{l}\text { Bat gemycircularvirus } \\
\text { Tibet } 2013\end{array}$ & $\begin{array}{r}\text { BtRh-CV-6 } \\
\text { Tibet2013 }\end{array}$ & China & $\begin{array}{l}\text { Rhinolophus } \\
\text { hipposideros }\end{array}$ & Bat & $\begin{array}{l}\text { Pharyngeal \& } \\
\text { rectal swabs }\end{array}$ & {$[32]$} \\
\hline KJ938717 & $\begin{array}{l}\text { Caribou feces-associated } \\
\text { gemycircularvirus }\end{array}$ & FaGmV-13 & Canada & $\begin{array}{l}\text { Rangifer } \\
\quad \text { tarandus }\end{array}$ & Caribou & Faeces & [21] \\
\hline KM510192 & $\begin{array}{l}\text { Bromus-associated circular } \\
\text { DNA virus } 3\end{array}$ & BasCV-3 & $\begin{array}{l}\text { New } \\
\text { Zealand }\end{array}$ & $\begin{array}{l}\text { Bromus } \\
\quad \text { hordeaceus }\end{array}$ & $\begin{array}{r}\text { Soft brome/ } \\
\text { Bull grass }\end{array}$ & Leaf & [13] \\
\hline KM598385 & $\begin{array}{l}\text { Odonata associated } \\
\text { gemycircularvirus-1 }\end{array}$ & OdaGmV-1 & USA & Ischnura posita & Damselfly & Abdomen & {$[6]$} \\
\hline
\end{tabular}


Table 1 continued

\begin{tabular}{|c|c|c|c|c|c|c|c|}
\hline $\begin{array}{l}\text { GenBank } \\
\text { accession } \\
\text { no. }\end{array}$ & Virus/putative virus name & Acronym & Country & Isolation source & $\begin{array}{l}\text { Common } \\
\text { name }\end{array}$ & Sample type & Reference \\
\hline KM598386 & $\begin{array}{l}\text { Odonata associated } \\
\text { gemycircularvirus-1 }\end{array}$ & OdaGmV-1 & USA & $\begin{array}{l}\text { Pantala } \\
\text { hymenaea }\end{array}$ & Dragonfly & Abdomen & {$[6]$} \\
\hline KM598387 & $\begin{array}{l}\text { Odonata associated } \\
\text { gemycircularvirus-2 }\end{array}$ & OdaGmV-2 & USA & $\begin{array}{l}\text { Aeshna } \\
\text { multicolor }\end{array}$ & Dragonfly & Abdomen & [6] \\
\hline KM598388 & $\begin{array}{l}\text { Odonata associated } \\
\text { gemycircularvirus-2 }\end{array}$ & OdaGmV-2 & USA & $\begin{array}{l}\text { Libellula } \\
\text { saturata }\end{array}$ & Dragonfly & Abdomen & [6] \\
\hline KM821747 & $\begin{array}{l}\text { Sewage-associated } \\
\text { gemycircularvirus-1 }\end{array}$ & SaGmV-1 & $\begin{array}{l}\text { New } \\
\text { Zealand }\end{array}$ & $\begin{array}{l}\text { Sewage } \\
\text { oxidation pond }\end{array}$ & - & Sewage & {$[12]$} \\
\hline KP133075 & Gemycircularvirus SL1 & GemyCV-SL1 & Sri Lanka & Homo sapiens & Human & $\begin{array}{l}\text { Cerebrospinal } \\
\text { fluid }\end{array}$ & [24] \\
\hline KP133076 & Gemycircularvirus SL2 & GemyCV-SL2 & Sri Lanka & Homo sapiens & Human & $\begin{array}{l}\text { Cerebrospinal } \\
\text { fluid }\end{array}$ & [24] \\
\hline KP133077 & Gemycircularvirus SL3 & GemyCV-SL3 & Sri Lanka & Homo sapiens & Human & $\begin{array}{l}\text { Cerebrospinal } \\
\text { fluid }\end{array}$ & {$[24]$} \\
\hline KP133078 & Gemycircularvirus BZ1 & GemyCV-BZ1 & Brazil & Homo sapiens & Human & Faeces & {$[24]$} \\
\hline KP133079 & Gemycircularvirus BZ2 & GemyCV-BZ2 & Brazil & Homo sapiens & Human & Faeces & [24] \\
\hline KP133080 & Gemycircularvirus NP & GemyCV-NP & Nepal & $\begin{array}{l}\text { Untreated } \\
\text { sewage }\end{array}$ & - & Sewage & [24] \\
\hline KP263543 & $\begin{array}{l}\text { Badger faeces-associated } \\
\text { gemycircularvirus }\end{array}$ & BafaGM588 & Portugal & Meles meles & $\begin{array}{c}\text { European } \\
\text { badger }\end{array}$ & Faeces & {$[4]$} \\
\hline KP263544 & $\begin{array}{l}\text { Mongoose feces-associated } \\
\text { gemycircularvirus a }\end{array}$ & MoFaGmV181a & Portugal & $\begin{array}{l}\text { Herpestes } \\
\text { ichneumon }\end{array}$ & $\begin{array}{l}\text { Egyptian } \\
\text { mongoose }\end{array}$ & Faeces & {$[4]$} \\
\hline KP263545 & $\begin{array}{l}\text { Mongoose feces-associated } \\
\text { gemycircularvirus b }\end{array}$ & MoFaGmV160b & Portugal & $\begin{array}{l}\text { Herpestes } \\
\text { ichneumon }\end{array}$ & $\begin{array}{l}\text { Egyptian } \\
\text { mongoose }\end{array}$ & Faeces & {$[4]$} \\
\hline KP263546 & $\begin{array}{l}\text { Mongoose feces-associated } \\
\text { gemycircularvirus c }\end{array}$ & MoFaGmV541c & Portugal & $\begin{array}{l}\text { Herpestes } \\
\quad \text { ichneumon }\end{array}$ & $\begin{array}{l}\text { Egyptian } \\
\text { mongoose }\end{array}$ & Faeces & {$[4]$} \\
\hline KP263547 & $\begin{array}{l}\text { Mongoose feces-associated } \\
\text { gemycircularvirus d }\end{array}$ & MoFaGmV478d & Portugal & $\begin{array}{l}\text { Herpestes } \\
\text { ichneumon }\end{array}$ & $\begin{array}{l}\text { Egyptian } \\
\text { mongoose }\end{array}$ & Faeces & {$[4]$} \\
\hline KP987887 & Gemycircularvirus $\mathrm{C} 1 \mathrm{c}$ & $\mathrm{C} 1 \mathrm{c}$ & France & Homo sapiens & Human & Plasma & [29] \\
\hline KR912221 & $\begin{array}{l}\text { Gemycircularvirus } \\
\text { gemy-ch-rat1 }\end{array}$ & Gemy-ch-rat 1 & China & $\begin{array}{l}\text { Rattus } \\
\text { norvegicus }\end{array}$ & Rat & Blood & {$[17]$} \\
\hline KT253577 & $\begin{array}{l}\text { Poaceae associated } \\
\text { gemycircularvirus-1 }\end{array}$ & PaGmV-1 & Tonga & $\begin{array}{l}\text { Brachiaria } \\
\text { deflexa }\end{array}$ & Signalgrass & Leaf & [18] \\
\hline KT253578 & $\begin{array}{l}\text { Poaceae associated } \\
\text { gemycircularvirus-1 }\end{array}$ & PaGmV-1 & Tonga & $\begin{array}{l}\text { Brachiaria } \\
\text { deflexa }\end{array}$ & Signalgrass & Leaf & {$[18]$} \\
\hline KT253579 & $\begin{array}{l}\text { Poaceae associated } \\
\text { gemycircularvirus-1 }\end{array}$ & $\mathrm{PaGmV}-1$ & Tonga & $\begin{array}{l}\text { Saccharum } \\
\text { hybrid }\end{array}$ & Sugarcane & Leaf & {$[18]$} \\
\hline KT309029 & $\begin{array}{l}\text { Poecile atricapillus GI tract- } \\
\text { associated gemycircularvirus }\end{array}$ & Gitract & USA & $\begin{array}{l}\text { Poecile } \\
\quad \text { atricapillus }\end{array}$ & $\begin{array}{l}\text { Black-capped } \\
\text { chickadee }\end{array}$ & $\begin{array}{l}\text { Buccal and } \\
\text { cloacal swab }\end{array}$ & [9] \\
\hline KT598248 & $\begin{array}{l}\text { Soybean leaf-associated } \\
\text { gemycircularvirus } 1\end{array}$ & SlaGemV1 & USA & Glycine max & Soybean & Leaf & {$[20]$} \\
\hline KT732790 & $\begin{array}{l}\text { Pacific flying fox } \\
\text { faeces associated } \\
\text { gemycircularvirus-1 }\end{array}$ & PfffaGmV-1 & Tonga & $\begin{array}{l}\text { Pteropus } \\
\text { tonganus }\end{array}$ & Bat & Faeces & [19] \\
\hline KT732791 & $\begin{array}{l}\text { Pacific flying fox } \\
\text { faeces associated } \\
\text { gemycircularvirus-1 }\end{array}$ & PfffaGmV-1 & Tonga & $\begin{array}{l}\text { Pteropus } \\
\text { tonganus }\end{array}$ & Bat & Faeces & [19] \\
\hline KT732792 & $\begin{array}{l}\text { Pacific flying fox } \\
\text { faeces associated } \\
\text { gemycircularvirus-2 }\end{array}$ & PfffaGmV-2 & Tonga & $\begin{array}{l}\text { Pteropus } \\
\text { tonganus }\end{array}$ & Bat & Faeces & [19] \\
\hline KT732793 & $\begin{array}{l}\text { Pacific flying fox } \\
\text { faeces associated } \\
\text { gemycircularvirus-2 }\end{array}$ & PfffaGmV-2 & Tonga & $\begin{array}{l}\text { Pteropus } \\
\text { tonganus }\end{array}$ & Bat & Faeces & [19] \\
\hline
\end{tabular}


Table 1 continued

\begin{tabular}{|c|c|c|c|c|c|c|c|}
\hline $\begin{array}{l}\text { GenBank } \\
\text { accession } \\
\text { no. }\end{array}$ & Virus/putative virus name & Acronym & Country & Isolation source & $\begin{array}{l}\text { Common } \\
\text { name }\end{array}$ & Sample type & Reference \\
\hline KT732794 & $\begin{array}{l}\text { Pacific flying fox } \\
\text { faeces associated } \\
\text { gemycircularvirus-3 }\end{array}$ & PfffaGmV-3 & Tonga & $\begin{array}{l}\text { Pteropus } \\
\text { tonganus }\end{array}$ & Bat & Faeces & [19] \\
\hline KT732795 & $\begin{array}{l}\text { Pacific flying fox } \\
\text { faeces associated } \\
\text { gemycircularvirus-4 }\end{array}$ & PfffaGmV-4 & Tonga & $\begin{array}{l}\text { Pteropus } \\
\text { tonganus }\end{array}$ & Bat & Faeces & [19] \\
\hline KT732796 & $\begin{array}{l}\text { Pacific flying fox } \\
\text { faeces associated } \\
\text { gemycircularvirus-4 }\end{array}$ & PfffaGmV-4 & Tonga & $\begin{array}{l}\text { Pteropus } \\
\text { tonganus }\end{array}$ & Bat & Faeces & [19] \\
\hline KT732797 & $\begin{array}{l}\text { Pacific flying fox } \\
\text { faeces associated } \\
\text { gemycircularvirus-5 }\end{array}$ & PfffaGmV-5 & Tonga & $\begin{array}{l}\text { Pteropus } \\
\text { tonganus }\end{array}$ & Bat & Faeces & [19] \\
\hline KT732798 & $\begin{array}{l}\text { Pacific flying fox } \\
\text { faeces associated } \\
\text { gemycircularvirus-6 }\end{array}$ & PfffaGmV-6 & Tonga & $\begin{array}{l}\text { Pteropus } \\
\text { tonganus }\end{array}$ & Bat & Faeces & [19] \\
\hline KT732799 & $\begin{array}{l}\text { Pacific flying fox } \\
\text { faeces associated } \\
\text { gemycircularvirus-6 }\end{array}$ & PfffaGmV-6 & Tonga & $\begin{array}{l}\text { Pteropus } \\
\text { tonganus }\end{array}$ & Bat & Faeces & [19] \\
\hline KT732800 & $\begin{array}{l}\text { Pacific flying fox } \\
\text { faeces associated } \\
\text { gemycircularvirus-7 }\end{array}$ & PfffaGmV-7 & Tonga & $\begin{array}{l}\text { Pteropus } \\
\text { tonganus }\end{array}$ & Bat & Faeces & [19] \\
\hline KT732801 & $\begin{array}{l}\text { Pacific flying fox } \\
\text { faeces associated } \\
\text { gemycircularvirus- } 8\end{array}$ & PfffaGmV-8 & Tonga & $\begin{array}{l}\text { Pteropus } \\
\text { tonganus }\end{array}$ & Bat & Faeces & [19] \\
\hline KT732802 & $\begin{array}{l}\text { Pacific flying fox } \\
\text { faeces associated } \\
\text { gemycircularvirus-8 }\end{array}$ & PfffaGmV-8 & Tonga & $\begin{array}{l}\text { Pteropus } \\
\text { tonganus }\end{array}$ & Bat & Faeces & [19] \\
\hline KT732803 & $\begin{array}{l}\text { Pacific flying fox } \\
\text { faeces associated } \\
\text { gemycircularvirus-9 }\end{array}$ & PfffaGmV-9 & Tonga & $\begin{array}{l}\text { Pteropus } \\
\quad \text { tonganus }\end{array}$ & Bat & Faeces & [19] \\
\hline KT732804 & $\begin{array}{l}\text { Pacific flying fox } \\
\text { faeces associated } \\
\text { gemycircularvirus-10 }\end{array}$ & PfffaGmV-10 & Tonga & $\begin{array}{l}\text { Pteropus } \\
\quad \text { tonganus }\end{array}$ & Bat & Faeces & [19] \\
\hline KT732805 & $\begin{array}{l}\text { Pacific flying fox } \\
\text { faeces associated } \\
\text { gemycircularvirus-10 }\end{array}$ & PfffaGmV-10 & Tonga & $\begin{array}{l}\text { Pteropus } \\
\quad \text { tonganus }\end{array}$ & Bat & Faeces & [19] \\
\hline KT732807 & $\begin{array}{l}\text { Pacific flying fox } \\
\text { faeces associated } \\
\text { gemycircularvirus-11 }\end{array}$ & PfffaGmV-11 & Tonga & $\begin{array}{l}\text { Pteropus } \\
\quad \text { tonganus }\end{array}$ & Bat & Faeces & [19] \\
\hline KT732808 & $\begin{array}{l}\text { Pacific flying fox } \\
\text { faeces associated } \\
\text { gemycircularvirus-11 }\end{array}$ & PfffaGmV-11 & Tonga & $\begin{array}{l}\text { Pteropus } \\
\quad \text { tonganus }\end{array}$ & Bat & Faeces & [19] \\
\hline KT732809 & $\begin{array}{l}\text { Pacific flying fox } \\
\text { faeces associated } \\
\text { gemycircularvirus-11 }\end{array}$ & PfffaGmV-11 & Tonga & $\begin{array}{l}\text { Pteropus } \\
\quad \text { tonganus }\end{array}$ & Bat & Faeces & [19] \\
\hline KT732810 & $\begin{array}{l}\text { Pacific flying fox } \\
\text { faeces associated } \\
\text { gemycircularvirus-11 }\end{array}$ & PfffaGmV-11 & Tonga & $\begin{array}{l}\text { Pteropus } \\
\quad \text { tonganus }\end{array}$ & Bat & Faeces & [19] \\
\hline KT732811 & $\begin{array}{l}\text { Pacific flying fox } \\
\text { faeces associated } \\
\text { gemycircularvirus-11 }\end{array}$ & PfffaGmV-11 & Tonga & $\begin{array}{l}\text { Pteropus } \\
\quad \text { tonganus }\end{array}$ & Bat & Faeces & [19] \\
\hline KT732812 & $\begin{array}{l}\text { Pacific flying fox } \\
\text { faeces associated } \\
\text { gemycircularvirus-11 }\end{array}$ & PfffaGmV-11 & Tonga & $\begin{array}{l}\text { Pteropus } \\
\quad \text { tonganus }\end{array}$ & Bat & Faeces & [19] \\
\hline
\end{tabular}


Table 1 continued

\begin{tabular}{|c|c|c|c|c|c|c|c|}
\hline $\begin{array}{l}\text { GenBank } \\
\text { accession } \\
\text { no. }\end{array}$ & Virus/putative virus name & Acronym & Country & Isolation source & $\begin{array}{l}\text { Common } \\
\text { name }\end{array}$ & Sample type & Reference \\
\hline KT732813 & $\begin{array}{l}\text { Pacific flying fox } \\
\text { faeces associated } \\
\text { gemycircularvirus-12 }\end{array}$ & PfffaGmV-12 & Tonga & $\begin{array}{l}\text { Pteropus } \\
\text { tonganus }\end{array}$ & Bat & Faeces & [19] \\
\hline KT732814 & $\begin{array}{l}\text { Pacific flying fox } \\
\text { faeces associated } \\
\text { gemycircularvirus-13 }\end{array}$ & PfffaGmV-13 & Tonga & $\begin{array}{l}\text { Pteropus } \\
\quad \text { tonganus }\end{array}$ & Bat & Faeces & [19] \\
\hline KT732806 & $\begin{array}{l}\text { Pacific flying fox } \\
\text { faeces associated } \\
\text { gemycircularvirus-14 }\end{array}$ & PfffaGmV-14 & Tonga & $\begin{array}{l}\text { Pteropus } \\
\quad \text { tonganus }\end{array}$ & Bat & Faeces & [19] \\
\hline KT862238 & $\begin{array}{l}\text { Faecal-associated } \\
\text { gemycircularvirus-14 }\end{array}$ & FaGmV-14 & $\begin{array}{l}\text { New } \\
\text { Zealand }\end{array}$ & $\begin{array}{l}\text { Anas } \\
\quad \text { platyrhynchos }\end{array}$ & Duck & Faeces & [28] \\
\hline KT862239 & $\begin{array}{l}\text { Faecal-associated } \\
\text { gemycircularvirus-14 }\end{array}$ & FaGmV-14 & $\begin{array}{l}\text { New } \\
\text { Zealand }\end{array}$ & $\begin{array}{l}\text { Anas } \\
\text { platyrhynchos }\end{array}$ & Duck & Faeces & [28] \\
\hline KT862240 & $\begin{array}{l}\text { Sewage-associated } \\
\text { gemycircularvirus-3 }\end{array}$ & $\mathrm{SaGmV}-3$ & $\begin{array}{l}\text { New } \\
\text { Zealand }\end{array}$ & $\begin{array}{c}\text { Gallus gallus } \\
\text { domesticus }\end{array}$ & Chicken & Faeces & {$[28]$} \\
\hline KT862241 & $\begin{array}{l}\text { Faecal-associated } \\
\text { gemycircularvirus-4 }\end{array}$ & $\mathrm{FaGmV}-4$ & $\begin{array}{l}\text { New } \\
\text { Zealand }\end{array}$ & $\begin{array}{c}\text { Gallus gallus } \\
\text { domesticus }\end{array}$ & Chicken & Faeces & [28] \\
\hline KT862242 & $\begin{array}{l}\text { Faecal-associated } \\
\text { gemycircularvirus-17 }\end{array}$ & FaGmV-17 & $\begin{array}{l}\text { New } \\
\text { Zealand }\end{array}$ & $\begin{array}{l}\text { Gallus gallus } \\
\text { domesticus }\end{array}$ & Chicken & Faeces & [28] \\
\hline KT862243 & $\begin{array}{l}\text { Faecal-associated } \\
\text { gemycircularvirus-20 }\end{array}$ & FaGmV-20 & $\begin{array}{l}\text { New } \\
\text { Zealand }\end{array}$ & $\begin{array}{c}\text { Gallus gallus } \\
\text { domesticus }\end{array}$ & Chicken & Faeces & [28] \\
\hline KT862244 & $\begin{array}{l}\text { Faecal-associated } \\
\text { gemycircularvirus-20 }\end{array}$ & FaGmV-20 & $\begin{array}{l}\text { New } \\
\text { Zealand }\end{array}$ & Lama glama & Llama & Faeces & [28] \\
\hline KT862245 & $\begin{array}{l}\text { Faecal-associated } \\
\text { gemycircularvirus-21 }\end{array}$ & FaGmV-21 & $\begin{array}{l}\text { New } \\
\text { Zealand }\end{array}$ & Lama glama & Llama & Faeces & [28] \\
\hline KT862246 & $\begin{array}{l}\text { Faecal-associated } \\
\text { gemycircularvirus-20 }\end{array}$ & FaGmV-20 & $\begin{array}{l}\text { New } \\
\text { Zealand }\end{array}$ & $\begin{array}{l}\text { Equus ferus } \\
\text { caballus }\end{array}$ & Horse & Faeces & [28] \\
\hline KT862247 & $\begin{array}{l}\text { Faecal-associated } \\
\text { gemycircularvirus-21 }\end{array}$ & FaGmV-21 & $\begin{array}{l}\text { New } \\
\text { Zealand }\end{array}$ & $\begin{array}{l}\text { Equus ferus } \\
\text { caballus }\end{array}$ & Horse & Faeces & [28] \\
\hline KT862248 & $\begin{array}{l}\text { Faecal-associated } \\
\text { gemycircularvirus-18 }\end{array}$ & FaGmV-18 & $\begin{array}{l}\text { New } \\
\text { Zealand }\end{array}$ & $\begin{array}{l}\text { Equus ferus } \\
\text { caballus }\end{array}$ & Horse & Faeces & [28] \\
\hline KT862249 & $\begin{array}{l}\text { Faecal-associated } \\
\text { gemycircularvirus-16 }\end{array}$ & FaGmV-16 & $\begin{array}{l}\text { New } \\
\text { Zealand }\end{array}$ & Ovis aries & Sheep & Faeces & [28] \\
\hline KT862250 & $\begin{array}{l}\text { Faecal-associated } \\
\text { gemycircularvirus-19 }\end{array}$ & FaGmV-19 & $\begin{array}{l}\text { New } \\
\text { Zealand }\end{array}$ & $\begin{array}{l}\text { Sus scrofa } \\
\quad \text { domestica }\end{array}$ & Pig & Faeces & [28] \\
\hline KT862251 & $\begin{array}{l}\text { Faecal-associated } \\
\text { gemycircularvirus-16 }\end{array}$ & FaGmV-16 & $\begin{array}{l}\text { New } \\
\text { Zealand }\end{array}$ & Ovis aries & Sheep & Faeces & [28] \\
\hline KT862252 & $\begin{array}{l}\text { Sewage-associated } \\
\text { gemycircularvirus-3 }\end{array}$ & $\mathrm{SaGmV}-3$ & $\begin{array}{l}\text { New } \\
\text { Zealand }\end{array}$ & Bos taurus & Cow & Faeces & [28] \\
\hline KT862253 & $\begin{array}{l}\text { Faecal-associated } \\
\quad \text { gemycircularvirus-22 }\end{array}$ & FaGmV-22 & $\begin{array}{l}\text { New } \\
\text { Zealand }\end{array}$ & Bos taurus & Cow & Faeces & [28] \\
\hline KT862254 & $\begin{array}{l}\text { Faecal-associated } \\
\text { gemycircularvirus-15 }\end{array}$ & FaGmV-15 & $\begin{array}{l}\text { New } \\
\text { Zealand }\end{array}$ & $\begin{array}{c}\text { Canis lupus } \\
\text { familiaris }\end{array}$ & Dog & Faeces & [28] \\
\hline KT862255 & $\begin{array}{l}\text { Sewage-associated } \\
\text { gemycircularvirus-3 }\end{array}$ & $\mathrm{SaGmV}-3$ & $\begin{array}{l}\text { New } \\
\text { Zealand }\end{array}$ & Lepus europaeus & Hare & Faeces & [28] \\
\hline LK931483 & HCBI8.215 virus & HCBI8_215 & Germany & Bos taurus & Cow & Serum & [16] \\
\hline LK931484 & HCBI9.212 virus & HCBI9_212 & Germany & Bos taurus & Cow & Serum & [16] \\
\hline LK931485 & MSSI2.225 virus & MSSI2_225 & Germany & Homo sapiens & Human & Blood & [16] \\
\hline
\end{tabular}


Fig. 2 Phylogenetic analysis of the replication-initiation proteins (Reps) of ssDNA viruses. The type species of the proposed genus

Gemycircularvirus within the family Genomoviridae is highlighted in boldface. For phylogenetic analysis, protein sequences were aligned using PROMALS3D [23], and columns containing gaps were removed from the alignment using trimAl (strict mode) [3]. Maximum-likelihood phylogenetic analysis was carried out using PhyML 3.1 [8] with the RtREV $+\mathrm{G} 6+\mathrm{I}+\mathrm{F}$ model, which was selected by PhyML as the best-fitting model. Numbers at the branch points represent aBayes local support values. Branches with lower than $60 \%$ support were collapsed. The scale bar represents the number of substitutions per site. For clarity, the tree was mid-point rooted. All taxa are indicated with the corresponding GenBank accession numbers, followed by abbreviated virus names (full virus names are provided in Table 1)

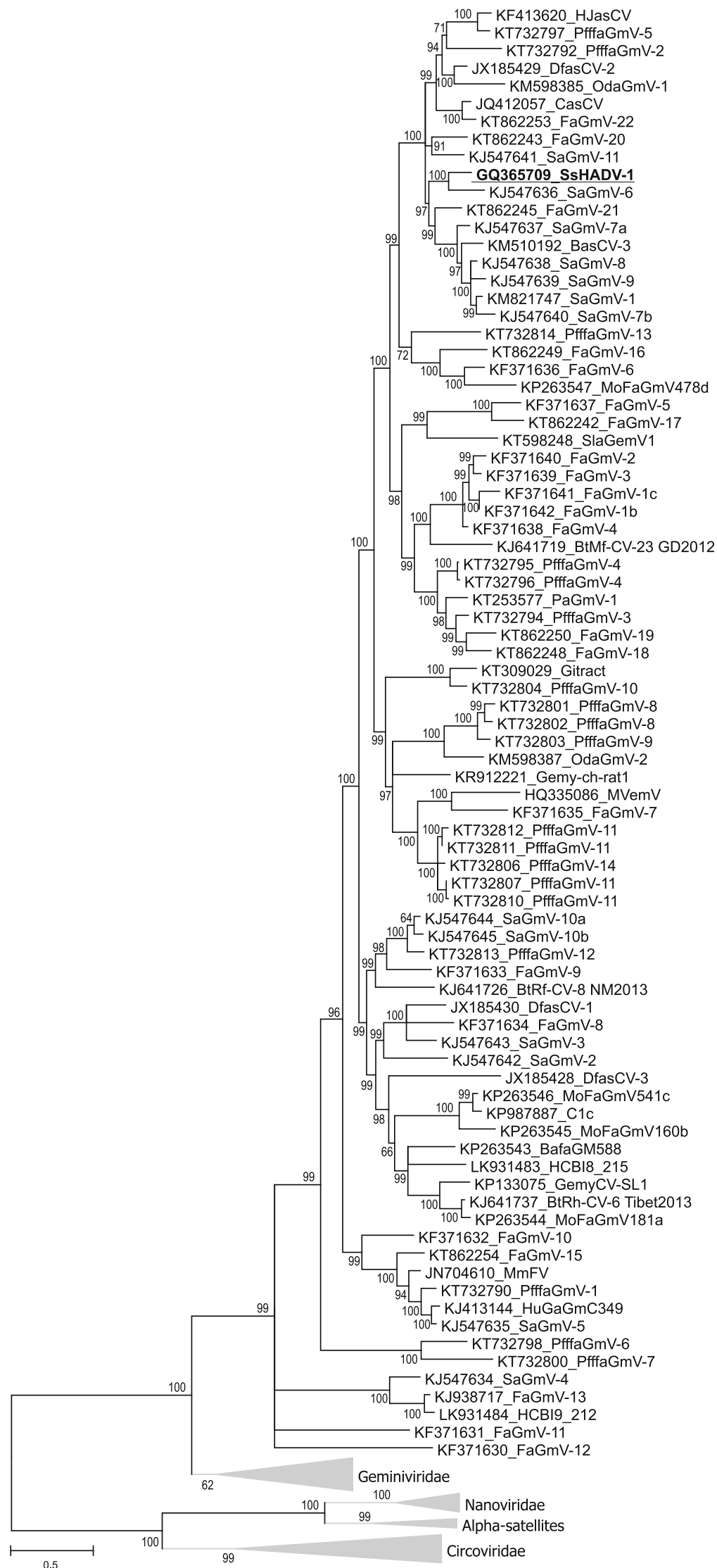

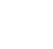

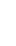

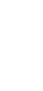


Fig. 3 Phylogenetic analysis of the SsHADV-1-like capsid proteins. The type species of the proposed genus

Gemycircularvirus within the family Genomoviridae is highlighted in boldface. For phylogenetic analysis, protein sequences were aligned using PROMALS3D [23], and columns containing gaps were removed from the alignment using trimAl (strictplus mode) [3]. Maximum-likelihood phylogenetic analysis was carried out using PhyML 3.1 [8] with the $\mathrm{LG}+\mathrm{G} 6+\mathrm{F}$ model, which was selected by PhyML as the best-fitting model. Numbers at the branch points represent aBayes local support values. Branches with lower than $60 \%$ support were collapsed. The scale bar represents the number of substitutions per site. The tree was rooted with the capsid proteins of geminiviruses. All taxa are indicated with the corresponding GenBank accession numbers, followed by abbreviated virus names (full virus names are provided in Table 1)

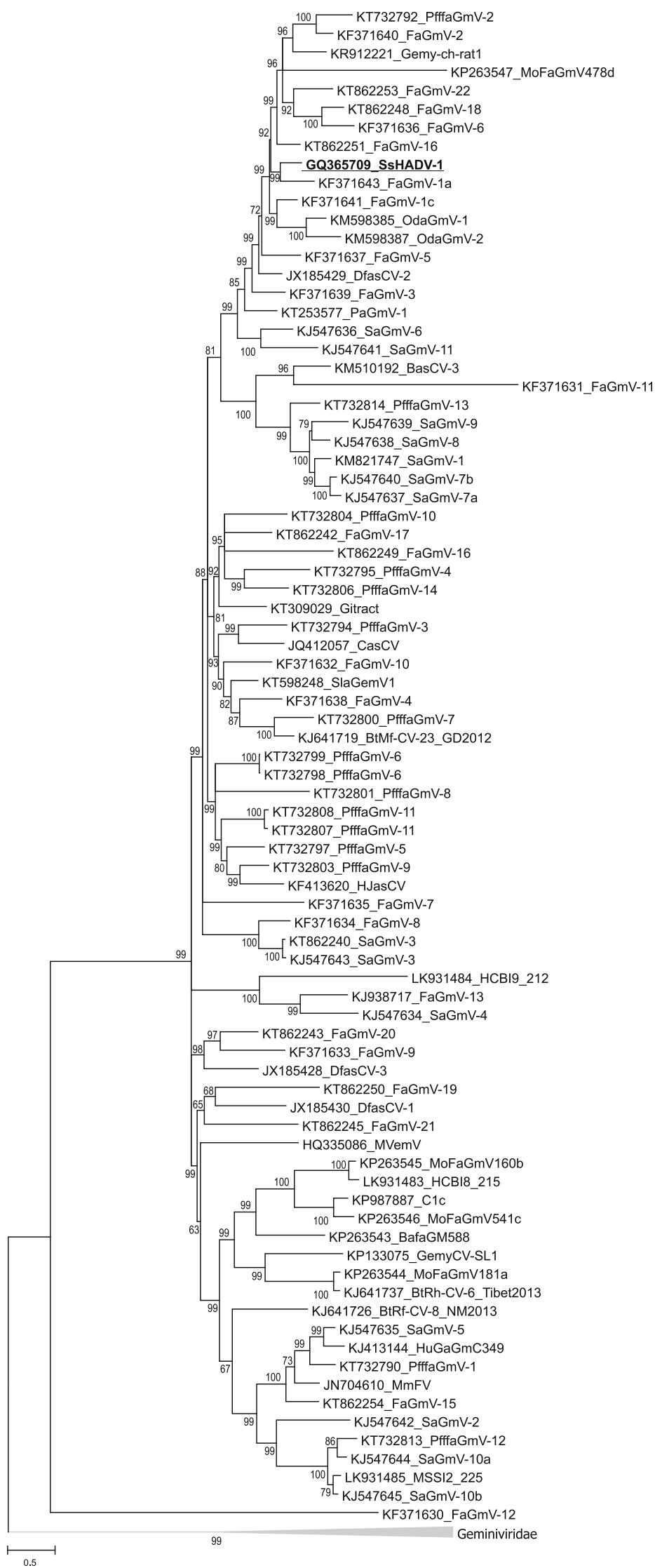




\section{Compliance with ethical standards}

The authors declare that no competing interests exist. This article does not contain any studies with human participants or animals performed by any of the authors.

\section{References}

1. Adams MJ, Lefkowitz EJ, King AMQ, Harrach B, Harrison RL, Knowles NJ, Kropinski AM, Krupovic M, Kuhn JH, Mushegian AR, Nibert M, Sabanadzovic S, Sanfaçon H, Siddell SG, Simmonds P, Varsani A, Zerbini FM, Gorbalenya AE, Davison AJ (2016) Ratification vote on taxonomic proposals to the International Committee on Taxonomy of Viruses (2016). Arch Virol (submitted)

2. Böttcher B, Unseld S, Ceulemans H, Russell RB, Jeske H (2004) Geminate structures of African cassava mosaic virus. J Virol 78:6758-6765

3. Capella-Gutierrez S, Silla-Martinez JM, Gabaldon T (2009) tri$\mathrm{mAl}$ : a tool for automated alignment trimming in large-scale phylogenetic analyses. Bioinformatics 25:1972-1973

4. Conceicao-Neto N, Zeller M, Heylen E, Lefrere H, Mesquita JR, Matthijnssens J (2015) Fecal virome analysis of three carnivores reveals a novel nodavirus and multiple gemycircularviruses. Virol J 12:79

5. Dayaram A, Opong A, Jaschke A, Hadfield J, Baschiera M, Dobson RC, Offei SK, Shepherd DN, Martin DP, Varsani A (2012) Molecular characterisation of a novel cassava associated circular ssDNA virus. Virus Res 166:130-135

6. Dayaram A, Potter KA, Pailes R, Marinov M, Rosenstein DD, Varsani A (2015) Identification of diverse circular single-stranded DNA viruses in adult dragonflies and damselflies (Insecta: Odonata) of Arizona and Oklahoma, USA. Infect Genet Evol 30:278-287

7. Du Z, Tang Y, Zhang S, She X, Lan G, Varsani A, He Z (2014) Identification and molecular characterization of a single-stranded circular DNA virus with similarities to Sclerotinia sclerotiorum hypovirulence-associated DNA virus 1. Arch Virol 159:1527-1531

8. Guindon S, Dufayard JF, Lefort V, Anisimova M, Hordijk W, Gascuel O (2010) New algorithms and methods to estimate maximum-likelihood phylogenies: assessing the performance of PhyML 3.0. Syst Biol 59:307-321

9. Hanna ZR, Runckel C, Fuchs J, DeRisi JL, Mindell DP, Van Hemert C, Handel CM, Dumbacher JP (2015) Isolation of a complete circular virus genome sequence from an Alaskan blackcapped chickadee (Poecile atricapillus) gastrointestinal tract sample. Genome Announc 3:01081-01015

10. Jiang D, Fu Y, Guoqing L, Ghabrial SA (2013) Viruses of the plant pathogenic fungus Sclerotinia sclerotiorum. Adv Virus Res $86: 215-248$

11. Kraberger S, Stainton D, Dayaram A, Zawar-Reza P, Gomez C, Harding JS, Varsani A (2013) Discovery of Sclerotinia sclerotiorum hypovirulence-associated virus-1 in urban river sediments of Heathcote and Styx Rivers in Christchurch City, New Zealand. Genome Announc 1:e00559-00513

12. Kraberger S, Arguello-Astorga GR, Greenfield LG, Galilee C, Law D, Martin DP, Varsani A (2015) Characterisation of a diverse range of circular replication-associated protein encoding DNA viruses recovered from a sewage treatment oxidation pond. Infect Genet Evol 31:73-86

13. Kraberger S, Farkas K, Bernardo P, Booker C, Arguello-Astorga GR, Mesleard F, Martin DP, Roumagnac P, Varsani A (2015)
Identification of novel Bromus- and Trifolium-associated circular DNA viruses. Arch Virol 160:1303-1311

14. Krupovic M (2013) Networks of evolutionary interactions underlying the polyphyletic origin of ssDNA viruses. Curr Opin Virol 3:578-586

15. Krupovic M, Forterre P (2015) Single-stranded DNA viruses employ a variety of mechanisms for integration into host genomes. Ann N Y Acad Sci 1341:41-53

16. Lamberto I, Gunst K, Muller H, Zur Hausen H, de Villiers EM (2014) Mycovirus-like DNA virus sequences from cattle serum and human brain and serum samples from multiple sclerosis patients. Genome Announc 2:e00848-00814

17. Li W, Gu Y, Shen Q, Yang S, Wang X, Wan Y, Zhang W (2015) A novel gemycircularvirus from experimental rats. Virus Genes 51:302-305

18. Male MF, Kami V, Kraberger S, Varsani A (2015) Genome sequences of Poaceae-associated Gemycircularviruses from the Pacific Ocean Island of Tonga. Genome Announc 3:e114401115

19. Male MF, Kraberger S, Stainton D, Kami V, Varsani A (2016) Cycloviruses, gemycircularviruses and other novel replicationassociated protein encoding circular viruses in Pacific flying fox (Pteropus tonganus) faeces. Infect Genet Evol 39:279-292

20. Marzano SL, Domier L (2016) Novel mycoviruses discovered from metatranscriptomics survey of soybean phyllosphere phytobiomes. Virus Res 213:332-342

21. Ng TF, Chen LF, Zhou Y, Shapiro B, Stiller M, Heintzman PD, Varsani A, Kondov NO, Wong W, Deng X, Andrews TD, Moorman BJ, Meulendyk T, MacKay G, Gilbertson RL, Delwart E (2014) Preservation of viral genomes in 700-y-old caribou feces from a subarctic ice patch. Proc Natl Acad Sci USA 111:16842-16847

22. Ng TFF, Willner DL, Lim YW, Schmieder R, Chau B, Nilsson C, Anthony S, Ruan YJ, Rohwer F, Breitbart M (2011) Broad surveys of DNA viral diversity obtained through viral metagenomics of mosquitoes. PLoS One 6:e20579

23. Pei J, Grishin NV (2014) PROMALS3D: multiple protein sequence alignment enhanced with evolutionary and three-dimensional structural information. Methods Mol Biol 1079:263-271

24. Phan TG, Mori D, Deng X, Rajindrajith S, Ranawaka U, Fan Ng TF, Bucardo-Rivera F, Orlandi P, Ahmed K, Delwart E (2015) Small circular single stranded DNA viral genomes in unexplained cases of human encephalitis, diarrhea, and in untreated sewage. Virology 482:98-104

25. Pietilä MK, Roine E, Sencilo A, Bamford DH, Oksanen HM (2016) Pleolipoviridae, a newly proposed family comprising archaeal pleomorphic viruses with single-stranded or doublestranded DNA genomes. Arch Virol 161:249-256

26. Rosario K, Dayaram A, Marinov M, Ware J, Kraberger S, Stainton D, Breitbart M, Varsani A (2012) Diverse circular ssDNA viruses discovered in dragonflies (Odonata: Epiprocta). J Gen Virol 93:2668-2681

27. Sikorski A, Massaro M, Kraberger S, Young LM, Smalley D, Martin DP, Varsani A (2013) Novel myco-like DNA viruses discovered in the faecal matter of various animals. Virus Res 177:209-216

28. Steel O, Kraberger S, Sikorski A, Young LM, Catchpole RJ, Steven AJ, Ladley JJ, Coray DS, Stainton D, Dayaram A, Julian L, van Bysterveldt K, Varsani A (2016) Circular replication-associated protein encoding DNA viruses identified in the faecal matter of various animals in New Zealand. Infect Genet Evol 43:151-164

29. Uch R, Fournier PE, Robert C, Blanc-Tailleur C, Galicher V, Barre R, Jordier F, de Micco P, Raoult D, Biagini P (2015) 
Divergent Gemycircularvirus in HIV-positive blood, France. Emerg Infect Dis 21:2096-2098

30. van den Brand JM, van Leeuwen M, Schapendonk CM, Simon JH, Haagmans BL, Osterhaus AD, Smits SL (2012) Metagenomic analysis of the viral flora of pine marten and European badger feces. J Virol 86:2360-2365

31. Varsani A, Navas-Castillo J, Moriones E, Hernandez-Zepeda C, Idris A, Brown JK, Murilo Zerbini F, Martin DP (2014) Establishment of three new genera in the family Geminiviridae: Becurtovirus, Eragrovirus and Turncurtovirus. Arch Virol 159:2193-2203

32. Wu Z, Yang L, Ren X, He G, Zhang J, Yang J, Qian Z, Dong J, Sun L, Zhu Y, Du J, Yang F, Zhang S, Jin Q (2015) Deciphering the bat virome catalog to better understand the ecological diversity of bat viruses and the bat origin of emerging infectious diseases. ISME J. doi:10.1038/ismej.2015.1138
33. Xie J, Jiang D (2014) New insights into mycoviruses and exploration for the biological control of crop fungal diseases. Annu Rev Phytopathol 52:45-68

34. Yu X, Li B, Fu Y, Jiang D, Ghabrial SA, Li G, Peng Y, Xie J, Cheng J, Huang J, Yi X (2010) A geminivirus-related DNA mycovirus that confers hypovirulence to a plant pathogenic fungus. Proc Natl Acad Sci USA 107:8387-8392

35. Yu X, Li B, Fu Y, Xie J, Cheng J, Ghabrial SA, Li G, Yi X, Jiang D (2013) Extracellular transmission of a DNA mycovirus and its use as a natural fungicide. Proc Natl Acad Sci USA 110:1452-1457

36. Zhang W, Olson NH, Baker TS, Faulkner L, Agbandje-McKenna M, Boulton MI, Davies JW, McKenna R (2001) Structure of the Maize streak virus geminate particle. Virology 279:471-477 